\title{
Estimation of Population Size and Dispersal Pattern of Sterile Male Aedes aegypti Using Mark-Release-Recapture (MRR) Technique
}

\author{
R. Zulfa ${ }^{1 *}$, S. Yuliawati ${ }^{1}$, M. Martini ${ }^{1}$, R. Hestiningsih ${ }^{1}$, B. Ernawan ${ }^{2}$ \\ ${ }^{I}$ Faculty of Public Health, Diponegoro Universiy, Jl. Profesor Soedarto, Tembalang, Semarang, Indonesia \\ ${ }^{2}$ Center for Isotopes and Radiation Application, National Nuclear Energy Agency (BATAN), \\ Jl. Lebak Bulus Raya No. 49, Jakarta 12440, Indonesia
}

\section{ARTICLE INFO}

\section{Article history:}

Received 30 April 2020

Received in revised form 30 April 2021

Accepted 30 April 2021

\section{Keywords:}

Sterile Insect Technique (SIT)

Ae. aegypti

Mark-Release-Recapture (MRR)

Dispersal

\begin{abstract}
A B S T R A C T
Aedes aegypti is currently emerging as a main vector of Dengue, Zika, and Chikungunya transmission. Chemical control was reported to be less effective due to the resistance of this mosquito to some types of insecticides. Therefore, another vector control is needed which is most appropriate to be used, i.e. the sterile insect technique (SIT). Information about optimum range dispersal sterile male Aedes aegypti for optimalization SIT program are needed. This study was designed to determine the dispersal pattern and population estimation of Aedes aegypti sterilized with gamma rays using mark-release-recapture (MRR) method. After the male Aedes aegypti (pupal stage) was irradiated with 70 Gy of gamma rays, the mosquitoes were then marked with Rhodamine-B and released into the study site. MRR experiments were carried out in Batan Indah residential area, and the Aedes aegypti were released in center of the site. Mosquitoes were recaptured at 28 points spread over the Batan Indah Residence for 2, 4, 6, and 8 days after release by using BG-Sentinel Traps. The result showed that the population of Aedes aegypti in the site was estimated to be 5.402 (1.347-14.636; CI 95\%) with the furthest spread distance was 119 meters from the release point. This study also showed that the MRR experiment can be used to estimate the population size and dispersal pattern of Aedes aegypti movement in a given locality. The result of present study provide better understanding for optimalization mosquito-borne disease prevention based on SIT programs.
\end{abstract}

(C) 2021 Atom Indonesia. All rights reserved

\section{INTRODUCTION}

Vector-borne disease become one of critical issues arise worldwide which causes more than 700.000 deaths per year. Vector-borne disease contribute more then $17 \%$ among all of the infectious disease, especially high prevalence on poorest population [1]. Outbreaks of vector-borne disease especially Dengue and Zika, which is transmitted by Aedes aegypti (Ae. aegypti), and related infectious disease have been occurring in tropical and subtropical regions, currently has been seen to be invasive into other areas [1]. Due to absence of effective vaccines, reducing the transmission cycles of the virus and vector

\footnotetext{
*Corresponding author.

E-mail address: Ramdanfkmundip2016@gmail.com

DOI: https://doi.org/10.17146/aij.2021.1052
}

abundance in urban areas are needed [2]. Nowdays, Transovarial phenomenon also being a big concern specially in dengue transmission. Based on study conducted by Martini et. al, transovarial was occurred in some city in Indonesia [3].

Insecticidal based control previously become the most reliable approach to suppress Ae. aegypti abundance. It has been reported to show less effectiveness due to resistance [4].

The practice of rearing and releasing mosquitoes for population control is gaining popularity as a novel approach with the potential to successfully control Ae. aegypti, the main vector of Dengue, Chikungunya, and Zika diseases $[5,6]$. Initiatives involving sequential inundative releases of mass-produced male mosquitoes using the sterile insect technique (SIT) by irradiation and/or the incompatible insect technique (IIT) are currently 
being planned and/or implemented in a number of countries [7-10]. However, elimination requires maintaining high sterile to wild male ratios that prove prohibitively costly [11].

SIT trials have been conducted and shown positive result in several study. The use of SIT has been carried out in several areas such as in Rowosari, Jangli and Ngalian areas in Semarang, Salatiga, Banjarnegara and West Bangka. The result of study in Jangli Neighborhood II showed that there is no dengue cases after the SIT were applied [12]. Furthermore, study conducted by Kitayyapong et al. in Thailand reported significantly reduction of the mean egg hatch rate $(84 \%)$ and the mean of females mosquito per household $(97.3 \%)$ after 6 month of sterile male release compared to the control site [7].

Mosquito population size is a fundamental parameter that is difficult to estimate accurately. Mark-release-recapture (MRR) is the most direct approach for estimating population density, dispersion patterns, flight distances, and survival rates of vector mosquitoes, In addition, MRR method is a common and direct approach to estimating population size of mosquitoes in specific areas [13].

The fluorescent dye Rhodamine- $\mathrm{B}$ is a relatively new development in the use of fluorescent markers to stain male spermatophores in insects, and it has provided a quick and inexpensive way to study mating interactions [14]. Rhodamine-B staining of male sperm, seminal fluids, and body tissues provides field ecologists with a means to measure both movement and mating interactions. When excitated under $540 \mathrm{~nm}$ (maximum excitation) and $568 \mathrm{~nm}$ (maximum emission) light wavelengths, the dye produces a distinct bright red fluorescence that can be seen in 95 percent of female Ae. aegypti spermathecae after four days [14]. By assessing behavioral and ecological factors such as mating success, investigators may mark both male and female mosquitoes, assess key performance measures, and quickly infer the effectiveness of an intervention.

Previous studies of Ae. aegypti dispersal have focus on females due to their importance for virus transmission [15]. Meanwhile, new approaches of releasing sterile male Ae. aegypti have been focusing on better understanding in ecological dispersal $[16,17]$. Studies conducting SIT mostly done to decrease the Dengue vector abundance and Dengue transmission without knowing the optimal range of male SIT mosquitoes dispersal range, especially for Ae. aegypti. This present study aimed to investigate the population size and dispersal pattern of the sterile male Ae. aegypti in the field using MRR trials. The information of this study will contribute to increase the effectiveness of the SIT application against DHF vector, Ae. aegypti, in the field.

\section{EXPERIMENTAL METHODS}

\section{Study area}

The study site, Batan Indah Residence, is suburban residential area located in Kademangan subdistrict, Setu district, South Tangerang Municipality, Banten Province. This location was chosen as study site due to high prevalence of Dengue fever cases in South Tangerang Municipality [18] and was already set as capture site for monitoring mosquito population from previous program conducted by BATAN. The Batan Indah Residence has a land area of 32 hectares and consist of around 1.000 household. 28 of BG-Sentinel traps (Biogents, Germany) were installed in different point over the study site (Fig. 1).

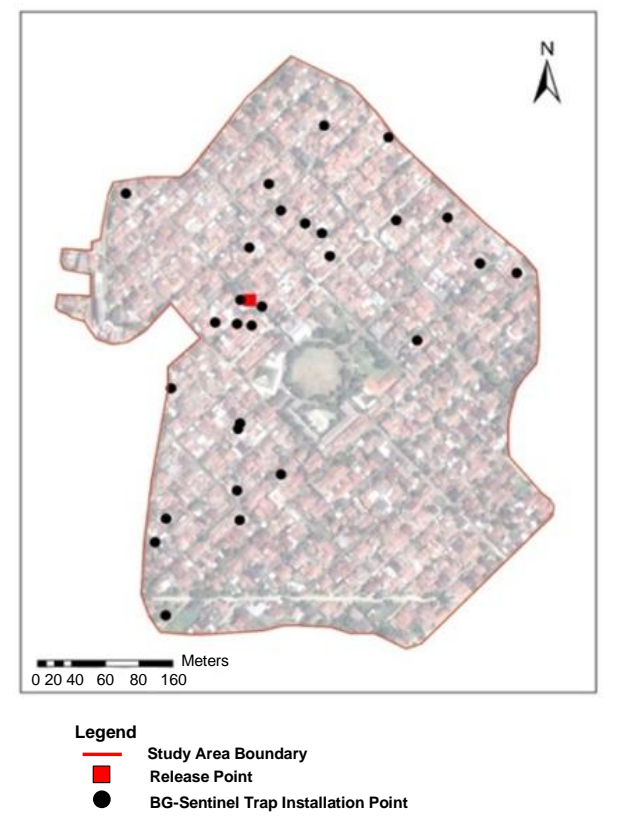

Fig. 1. Batan Indah site.

\section{Mosquitoes rearing and marking sterile male Ae. aegypti procedure}

The strains used this study was originally obtained from South Tangerang city, Banten Province and has been reared by Entomology Laboratory of CIRA BATAN since 2014. Mosquitoes were reared from hatching eggs using 40 of $30 \times 20 \times 10 \mathrm{~cm}$ trays filled with $\pm 5 \mathrm{~cm}$ of water depth. After the eggs hatched into larvae, they were fed using crushed dog biscuit (Pedigree ${ }^{\circledR}$ ) mixed with chicken liver with a ratio of $1: 1$, while the adult mosquitoes are kept in a $100 \times 40 \times 40 \mathrm{~cm}$ 
Bugdorm. In order to mantain the condition of the Ae. aegypti mass rearing, room temperature and humidity were recorded regularly everyday. After the larvae turned into pupae, the male and female pupae are separated using larval-pupa separator (John W. Hock Company, USA). Irradiation of the male pupae was conducted using the Gamma Cell 220 irradiator with Cobalt-60 as radioactive source. The gamma rays was applied with 70 Gy dosage based on the previous study that showed significant result of sterile males [19].

The marking technique of the sterile male Ae. aegypti was carried out using Rhodamine-B $0.4 \%$ when the pupae turned into adult stage. The procedure was done by mixing it into a $10 \%$ sugar solution and left to sit for 4 days. Finally, the sterile male Ae. aegypti were released into the study site.

\section{Sterile male Ae. aegypti release and recapture procedure}

A total of 5.000 marked sterile male mosquitoes were released in May 2019 in the study site. Then the mosquitoes were recaptured on the $2,4,6$, and 8 days post the release day.

The marked male mosquito is released by placing the Bugdorm at the release point and left it for 30 minutes to allow all sterile male mosquitoes inside the Bugdorm to come out.

Previously, the BG-Sentinel Trap with the BG-Lures attractant were used and installed at 28 home points to trap the mosquitoes, and the points of release were recorded using GPS. At each recapturing points, temperature and humidity at the time of collection were recorded (Fig. 1).

The population was estimated with the Fisher-Ford method, which is considered as the most accurate method to estimate the population size of a group. This method uses sensitive parameters of the daily survival probability of each individual. The principle of this method is to figure out the number of mosquitoes to be released and the number of mosquitoes that are recaptured from the environment. The formula used to calculate the population size estimation is the following Eq. (1) [20]:

$$
N=\left[\emptyset^{t} n \frac{M}{m}\right]-M
$$

Using correction formula by Fisher and Ford for low recapture $(<20)$ shown in Eq. (2) [21]:

$$
N=\left[\frac{\phi^{t}(n+1)(M+1)}{m+1}\right]-1-M
$$

Where: $N$ = estimation of population size

$M=$ marked mosquitoes release

$\varnothing=$ daily survival probability

$T$ = day between release and recapture

$N=$ number of mosquitoes capture

$M=$ number of mosquitoes recaptured

The confidence interval corresponds with the estimation calculated using the percentile bootstrap method, with 500 replication of $95 \%$ confidence interval percentage. For this research, the daily survival probabilty of Ae. aegypti mosquitoes is 0.65 based on a study conducted by Marco Neira et al. [22].

\section{RESULTS AND DISCUSSION \\ Estimation of Ae. aegypti population size}

Table 1 illustrates that the sterile male Ae. aegypti were only found on the $2^{\text {nd }}$ and $4^{\text {th }}$ day, 11 and 5 samples marked mosquitoes was recorded respectively. After calculating the data of the sterile male Ae. aegypti mosquito recapture using the Fisher-Ford population size estimation formula, the estimated Ae. aegypti mosquito population in Batan Indah residential area during this study was recorded at 5,402 $(\varnothing=0.65)(1,347-14,636, \mathrm{CI}=95 \%)$.

Table 1. Number of marked mosquitoes recaptured and capture on BG-sentinel based on day recaptured after marked mosquitoes release (day 0 ).

\begin{tabular}{cccc}
\hline \multirow{2}{*}{ Day } & \multicolumn{2}{c}{ Mosquitoes Captured } & \multirow{2}{*}{ Total } \\
\cline { 2 - 3 } & Marked & Unmarked & \\
\hline 2 & 11 & 111 & 122 \\
4 & 5 & 43 & 48 \\
6 & - & 35 & 35 \\
8 & - & 25 & 25 \\
\hline
\end{tabular}

In this study, the estimated population size of the $A e$. aegypti mosquitoes in the study site was recorded as 5,402 $(1,347-14,636, \mathrm{CI}=95 \%)$. This study was conducted during rainy season. However, based on study conducted by Tantowijoyo et. al. the population of Ae. aegypti mosquitoes, especially in Yogyakarta, tends to be stable throughout any season. Genetic variation is strongly affected by habitat. In habitat where the living spaces surrounded by natural barriers such as vegetation, genetic variation will be greater compared to urban habitats that are connected to each other [23]. Throughout the survey period, mosquitoes capture rates were significantly influenced by time of season and precipitation. In Matthew et al study, positive precipitation have been reported on the relative abundance of four most common mosquitoes species, include Ae. aegypti [24]. 
Furthermore, Ae. aegypti utilizes artificial and human-watered containers significantly for ovipositing, which could become more abundant related to heavy rainfall. The expansion of habitat for Ae. aegypti mosquitoes is caused by increased mobility and transportation from one region to another. As a consequence, it leads to the rising of the air temperature. Geographical expansion on the density of Ae. aegypti larvae and pupae may be related to water limitations, waste location, changes in foraging behavior, and changes in the way water is stored in houses. The population size estimation of the Ae. aegypti mosquito can be used to monitor the population density of the adult Ae. aegypti mosquitoes in an area. Hence, by knowing the estimated population size, we will be able to take earlier action in controlling its population.

\section{Dispersal pattern of sterile male Ae. aegypti}

The dispersal pattern of the sterile male Ae. aegypti is presented in Fig. 2. Recorded based on 28 BG-sentinel traps that were installed in Batan Indah. As shown in Fig. 2(a), the dispersal of the sterile Ae. aegypti male mosquito on the 2nd day, where the marked sterile male mosquito were recaptured by the 4 BG-Sentinel trap with 5 marked sterile male mosquitoes found at a distance of 11 meters from the release point, at least 1 marked male mosquito was found in the BG-Sentinel installed with a distance from the release point of 49 meters and 109 meters, and 4 mosquitoes caught in the BG-Sentinel trap which had a distance of 16 meters from the release point.

In Fig. 2(b), on the 4th day, the marked male mosquito was recaptured by the 4 BG-Sentinel trap. Each 1 mosquito was caught in the BG-Sentinel trap which had a distance from the release point as far as 33 meters, 65 meters, and 119 meters, respectively, and 2 mosquitoes that were caught in the BGSentinel trap were found with a distance of 16 meters from the release point. Figure $2(\mathrm{c})$ is an overall distribution pattern map of the research. The closest distance of the marked mosquitoes found is 11 meters from the point of release and the farthest distance is 119 meters.

This research found that the distribution pattern of the sterile male mosquitoes released in Batan Indah was scattered randomly in all directions with the farthest distance of 119 meters from the location of release. In contrast, study conducted by Kitayyapong et. al. in Thailand reported further length of dispersal distances which variying from $140-625 \mathrm{~m}$ from the release point [7].
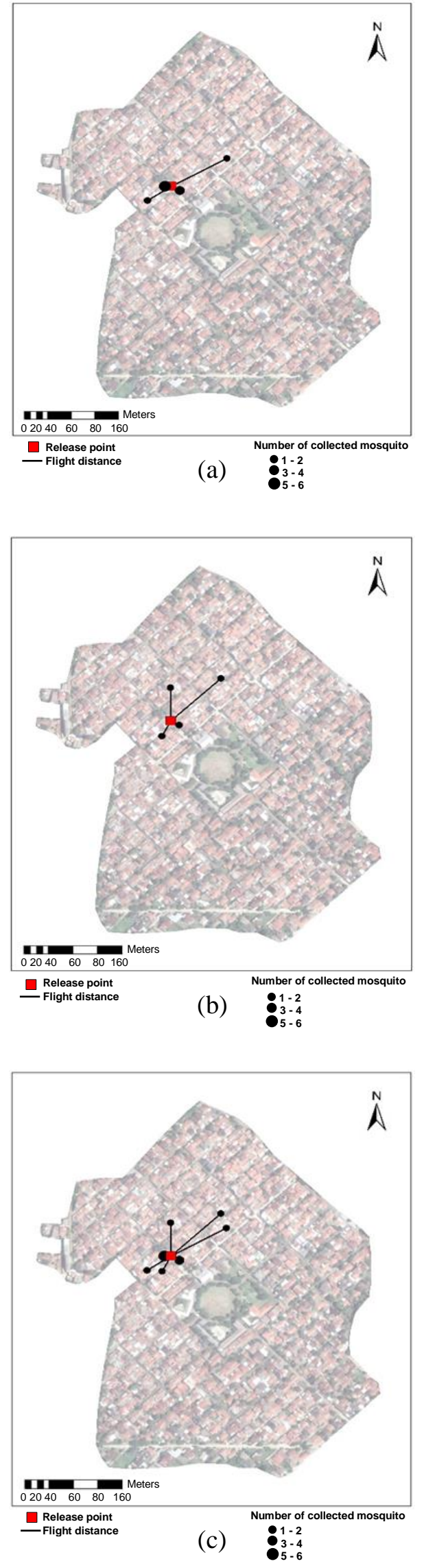

Fig. 2. (a) Dispersal pattern of sterile male Ae. aegypti mosquito $2^{\text {nd }}$ day after release, (b) Dispersal pattern of sterile male Ae. aegypti mosquito $4^{\text {th }}$ day after release, and (c) Dispersal pattern of sterile male Ae. aegypti mosquito during study. 
In support of this present study, several previous studies were recorded that MRR studies based on reared mosquitoes from laboratory generally showed limited dispersal [25]. Furthermore, this study was conducted in rainfall season and may affect the dispersal distance. Similar study was found that seasonal environmental condition contributes to the ecological plasticity of male Ae. aegypti [26].

The data on the dispersal pattern of the Ae. aegypti male mosquitoes will be very useful for the future application of SIT and as a benchmark to maximize the appliication due to predetermined bionomic distribution patterns of the sterile Ae. aegypti male mosquitoes. However, the limitation of this study is that it did not examine the longevity of sterile male Ae. aegypti and also did not include any environmental parameter (season, wind, precipitation and temperature) to ensure that the dispersal of males mosquitoes Ae. aegypti was valid.

\section{CONCLUSION}

Gamma rays 70 Gy was effectively produced sterile male mosquitoes. Recapture rate of sterile male Ae. aegypti was $0.32 \%$. The low number of sterile male Ae. aegypti mosquitoes recapture is possibly influenced by several factors such as the low life expectancy of sterile male Ae. aegypti mosquitoes and the influence of climate when conducting research, especially during sampling. The estimation of population size using MRR was $5.402(\varnothing=0.65)$ $(1,347-14,636$, CI $=95 \%)$, while the furthest distance of sterile male Ae. aegypti dispersal was 119 meters from the release point. The result of present study emphasizes for optimalization of mosquito-borne disease preventon based on SIT program to define adequate release point to achieve optimal result.

\section{ACKNOWLEDGMENT}

We acknowledge to the Center for Isotopes and Radiation Application, National Nuclear Energy Agency which has helped the implementation of this study and the supervisors who have helped compile this study.

\section{AUTHOR CONTRIBUTION}

Participated in research design: R. Zulfa, M. Martini and B. Ernawan, Conducted experiments:
$\mathrm{R}$. Zulfa, Wrote and contribute to the writing of the manuscript: R. Zulfa, M. Martini, S. Yuliawati, R. Hestiningsih and B. Ernawan.

All authors read and approved the final version of the paper.

\section{REFERENCES}

1. Anonymous, Vector-Borne Diseases. https://www.who.int/news-room/fact-sheets/ detail/vector-bornediseases. Retrieved in July (2020).

2. Anonymous, $C D C$, Surveillance and Control of Aedes aegypti and Aedes albopictus, in the United States. https://www.cdc.gov/chikungunya/pdfs/ surveillance-and-control-of-aedes-aegyptiandaedes-albopictus-us.pdf. Retrieved in August (2020)

3. C. L. Moyes, J. Vontas, A. J. Martins et al., PLoS Negl. Trop. Dis. 11 (2017) 1.

4. Anonymous, Evaluation of innovative strategies for Aedes aegypti control: challenges for their introduction and impact assessment, Pan American Health Organization, Washington, D.C (2019).

5. Anonymous, Guidance framework for testing the sterile insect technique as a vector control tool against Aedes-borne diseases, World Health Organization and the International Atomic Energy Agency, Geneva, Switzerland (2020).

6. P. Kittayapong, S. Ninphanomchai, W. Limophasmanee et al., Plos Negl. Trop. Dis. 13 (2019) 1.

7. J. W. Mains, P. H. Kelly, K. L. Dobson et al., J. Med. Entomol. 56 (2019) 1296.

8. J. Bouyer, H. Yamada, R. Pereira et al., Trends Parasitol. 36 (2020) 325.

9. J. E. Crawford, D. W. Clarke, V. Criswell et al., Nat. Biotechnol. 38 (2020) 482.

10. H. A. Flores, S. L. O’Neill, Nat. Rev. Microbiol. 16 (2018) 508.

11. D. Sutiningsih, A. Rahayu and D. Puspitasari,. J. Trop. Life Sci. 7 (2017) 133.

12. M. Q. Benedict, J. D. Charlwood, L. C. Harrington et al., Vector-Borne Zoonotic Dis. 18 (2018) 39.

13. B. J. Johnson, S. N. Mitchell, C. J. Paton et al., PLoS Negl. Trop. Dis. 11 (2017) e0005902.

14. C. A. Guerra, R. C. Reiner, T. A. Perkins et al., Parasites Vectors 7 (2014) 1. 
15. M. C. Medeiros, E. C. Boothe, E. B. Roark et al., PLoS Negl. Trop. Dis. 11 (2017) e0005347.

16. M. A. Opiyo, G. L. Hamer, D. W. Lwetoijera et al., PLoS One 11 (2016) 0159067.

17. Anonymous, South Tangerang in Figures, Statistic Department of South Tangerang City (2017).

18. B. Ernawan, U. S. F. Tambunan, I. Sugoro et al., Effects of Gamma Irradiation Dose-Rate on Sterile Male Aedesaegypti, Proceeding of International Biology Conference (2016).

19. W. K. Reisen, F. Mahmood and T. Parveen, Popul. Ecol. 21 (1979) 12.

20. N. T. J. Bailey, J. Anim. Ecol. 21 (1952) 120.
21. M. Niera, R. Lacroix, L. Caceres et al., Mem. Inst. Oswaldo Cruz. 109 (2014) 879.

22. W. Tantowijoyo, E. Arguni, P. Johnson et at., J. Med. Entomol. 53 (2016) 188.

23. V. J. Matthew, C. Brenda, R. J. Gregory et al., Parasites Vectors 13 (2020) 543.

24. L. Valerio, L. Facchinelli, J. M. Ramsey et al., Am. J. Trop. Med. Hyg. 86 (2012) 665.

25. J. G. Juarez, G. L. Selene, L. F. Chaves et al., Sci. Rep. 10 (2020) 6803.

26. M. Martini, A. Zufri, N. Kusariana et al., Entomological Status Based on Vector Density Index and Transovarial Infection on Aedes Sp. Mosquito in Meteseh Village, Semarang City, E3S Web of Conferences 202 (2020) 12016. 